\title{
PREOPERATIVE ASSESSMENT OF HAND CIRCULATION BY MEANS OF DOPPLER ULTRASONOGRAPHY AND THE MODIFIED ALLEN TEST
}

Permyos Ruengsakulrach, MD, FRCST ${ }^{\mathrm{a}}$ Mark Brooks, MB BS, FRACR ${ }^{\mathrm{b}}$

David L. Hare, MB, DPM, FRACP Ian Gordon, MSc, $\mathrm{PhD}^{\mathrm{d}}$

Brian F. Buxton, MS, FRACS
Objective: The aims of this study were as follows: (1) to evaluate Doppler ultrasonography in assessing hand collateral circulation; (2) to define the criteria for an abnormal Doppler ultrasonography dynamic test result; and (3) to validate the modified Allen test.

Methods: The hand circulation of 71 patients scheduled for coronary artery bypass grafting was assessed by means of the Allen test and Doppler ultrasonography. The flow in the superficial palmar branch of the radial artery, the ulnar artery, and the dorsal digital thumb artery with and without radial artery compression were recorded. Flow patterns in the superficial palmar branch of the radial artery, the ulnar artery, and the dorsal digital thumb artery with radial artery compression were categorized into 4 groups: (1) no flow; (2) decreased flow; (3) reversed flow; and (4) increased flow.

Results: Among the 71 hands, $4(5.6 \%)$ had an abnormal Allen test result (>10 seconds). Seven (10.6\%) of 66 superficial palmar branches of the radial artery, $3(4.2 \%)$ of 71 ulnar arteries, and $2(2.8 \%)$ of 71 dorsal digital thumb arteries showed no flow with radial artery compression, as measured by Doppler ultrasonography. There were significant differences among the 4 groups (superficial palmar branch of the radial artery: $\mathrm{F}=7.0, P<.001$; ulnar artery: $\mathrm{F}=13.1, P<.001$; and dorsal digital thumb artery: $\mathrm{F}=8.4$, $P<.001)$ for the Allen test. Pairwise comparisons showed that when subjected to an Allen test, category 1 patients (no flow) had significantly longer recovery times compared with the other groups $(P<.02$ in all cases $)$ for the superficial palmar branch of the radial artery, the ulnar artery, and the dorsal digital thumb artery.

Conclusion: Absence of flow in the dorsal digital thumb artery with radial artery compression is considered an absolute contraindication to radial artery harvesting. An increased recovery time with the modified Allen test predicts absence of flow in the dorsal digital thumb artery in Doppler ultrasonographic flow patterns. This demonstrates the validity of the modified Allen test for primary screening. (J Thorac Cardiovasc Surg 2001;121:526-31)
$\mathrm{W}^{\mathrm{i}}$ ith the resurgence of the use of the radial artery (RA) for coronary artery bypass grafting, tests such as Doppler ultrasonography and the Allen test have been increasingly used as screening devices for assessing the hand collateral circulation. ${ }^{1-3}$ The Allen test is a simple

From the Department of Cardiac Surgery, ${ }^{a}$ the Department of Radiology, ${ }^{\mathrm{b}}$ the Department of Cardiology, ${ }^{\mathrm{c}}$ Austin \& Repatriation Medical Centre, and the Department of Mathematics and Statistics ${ }^{\mathrm{d}}$ the University of Melbourne, Victoria, Australia.

Copyright (ㄷ 2001 by The American Association for Thoracic Surgery

0022-5223/2001 $\$ 35.00+0 \quad \mathbf{1 2 / 1 / 1 1 2 4 6 8}$

doi: $10.1067 / \mathrm{mtc} .2001 .112468$ and cost-effective test. However, there is still some debate about whether it is a valid screening test. Doppler ultrasonography has a number of benefits. However, one of the drawbacks of using this test is that there are no established standard criteria for differentiating between normal and abnormal Doppler ultrasonographic results. Several published studies have attempted to establish a standard set of criteria. $^{4-10}$ All studies have agreed on the need for a dynamic component comparing Doppler ultrasonographic signals with and without compression of the RA. However, the criteria for an abnormal result vary from study to study, depending on which vessels are scanned.

The specific aims of this study were as follows: (1) to evaluate Doppler ultrasonography in assessing the hand 
Table I. The distribution flow patterns in the UA, the SPA, and the TA

\begin{tabular}{lccc}
\hline $\begin{array}{l}\text { Changes in peak systolic } \\
\text { velocity with RA compression }\end{array}$ & UA flow pattern, $n(\%)$ & SPA flow pattern, $n(\%)$ & TA flow pattern, $n(\%)$ \\
\hline No flow & $3(4.2)$ & $7(10.6)$ & $2(2.8)$ \\
Decreased flow & $13(18.3)$ & $26(39.4)$ & $61(85.9)$ \\
Reversed flow & 0 & $18(27.3)$ & $2(2.8)$ \\
Increased flow & $54(76.1)$ & $15(22.7)$ & $6(8.5)$ \\
Total & $71(100)$ & $66(100)$ & $71(100)$ \\
\hline
\end{tabular}

collateral circulation; (2) to establish the criteria for defining an abnormal Doppler ultrasonography dynamic test result; and (3) to validate the modified Allen test.

\section{Methods}

Study patients. Seventy-one patients (59 men and 12 women; mean age, $67.3 \pm 9.8$ years) who were eligible for coronary artery bypass grafting between July 1998 and May 1999 entered into this study. Informed consent was obtained. The study was approved by the Austin \& Repatriation Medical Centre Ethics Committee. The nondominant hands of patients were tested with both the modified Allen test and Doppler ultrasonography. All observers were blinded to the results of the other tests. In terms of the characteristics of the patients, 9.9\% (7/71) were active smokers, 31\% (22/71) were diabetic, 30\% (21/71) had peripheral vascular disease, and $72 \%$ (51/71) had hypercholesterolemia.

\section{Testing methods}

Modified Allen test. The modified Allen test was performed according to the following protocol. The examiner faces the patient, whose hand is supinated. The RA and ulnar artery (UA) are located by their pulses. The examiner places each thumb lightly over the RA and UA simultaneously, with the 4 fingers of each hand placed behind the patient's wrist, thus holding the wrist lightly between the thumb and fingers. The patient's hand is closed as tightly as possible for a period of 1 minute. The patient is then asked to relax the hand and extend the fingers into a slightly flexed position while the examiner maintains pressure on the RA and UA. The hand at this point should appear blanched. The examiner then releases the pressure on the UA and continues applying pressure to the RA. The return of color to the hand and fingers is noted. In our study the recovery time was recorded as the time taken (in seconds) for the hand to return to its normal color after the release of the UA. One experienced observer performed all of the tests. An abnormal modified Allen test result was defined as a recovery time of more than 10 seconds.

Doppler ultrasonography dynamic test. Doppler ultrasonographic examination was performed the day before the operation by one of two sonographers using an Advanced Technology Laboratories (Bothell, Wash) HDI 5000 system with a $10-\mathrm{MHz}$ compact linear array transducer. The temperature in the ultrasound laboratory was maintained between a range of $23^{\circ} \mathrm{C}$ and $25^{\circ} \mathrm{C}$. Patients sat comfortably on a chair, with their forearms and hands positioned at the level of the heart.
Ultrasonography was used to identify the bifurcation of the brachial artery, the RA, and the UA in the forearm. A high division of the brachial artery was recorded. We then examined the following: (1) the UA at the wrist; (2) the superficial palmar branch of the RA (SPA) arising from the distal RA on the palmar aspect; and (3) the dorsal digital thumb artery (TA) located at the medial side of the base of the thumb. The Doppler probe was placed over the expected anatomic position of the artery and aligned with it at an angle of less than $60^{\circ}$. By moving the probe from side to side across the vessel, the center of the artery could be located readily by a characteristic noise produced at the frequency of the pulse rate. When a steady state was achieved, the RA was firmly compressed. The peak systolic velocity of flow in the UA, SPA, and TA with and without RA compression was recorded. To ensure a consistent sample volume, the sonographers attempted to keep the Doppler tracing on the same screen before and after RA compression while the peak systolic velocity was recorded.

Flow patterns in the UA were categorized into 3 groups: (1) no flow; (2) decreased flow; and (3) increased flow. Flow patterns in the SPA and TA with RA compression were categorized into 4 groups: (1) no flow; (2) decreased flow; (3) reversed flow; and (4) increased flow.

Statistical analysis. Data analysis was carried out by using SPSS PC version 10 software (SPSS, Inc, Chicago, Ill). The data were summarized in terms of a mean and SD. Analysis of variance was used to compare the mean differences of the recovery time by using the modified Allen test among the 4 categories of flow patterns in the SPA, UA, and TA. The recovery time with the modified Allen test was used as a single continuous response variable. Tests of normality were carried out, and where necessary, Box-Cox transformations were used to obtain scales for which the assumptions of the analysis of variance were justified. The Tukey multiple comparisons procedure was used to test for pairwise differences between the flow patterns in the UA, SPA, and TA groups.

Standard estimates of sensitivity, specificity, and positive and negative predictive value were obtained by using a $2 \times 2$ contingency table.

\section{Results}

Forearm. Ultrasonography identified a high division of the brachial artery in $5.6 \%$ (4/71) of the patients. All of the UAs and RAs were patent at the bifurcation and the elbow. 
Table II. Comparison of the modified Allen test and Doppler ultrasonography dynamic test in the UA, the SPA, and the TA flow pattern groups

\begin{tabular}{|c|c|c|c|c|c|c|c|}
\hline & & \multicolumn{2}{|c|}{ UA flow } & \multicolumn{2}{|c|}{ SPA flow } & \multicolumn{2}{|c|}{ TA flow } \\
\hline & & Absent & Present & Absent & Present & Absent & Present \\
\hline Allen test & $>10 \mathrm{~s}$ & 2 & 2 & 2 & 2 & 2 & 2 \\
\hline (recovery time) & $0-10 \mathrm{~s}$ & 1 & 66 & 5 & 57 & 0 & 67 \\
\hline Total & & 3 & 68 & 7 & 59 & 2 & 69 \\
\hline
\end{tabular}

Present, includes increased, decreased, and reversed flow patterns.

Table III. Test characteristics of the modified Allen test compared with the Doppler ultrasonography dynamic test

\begin{tabular}{|c|c|c|c|c|c|c|}
\hline \multirow[b]{2}{*}{ Characteristic } & \multicolumn{2}{|c|}{ UA flow } & \multicolumn{2}{|c|}{ SPA flow } & \multicolumn{2}{|c|}{ TA flow } \\
\hline & $\%$ & $95 \% C I$ & $\%$ & $95 \% C I$ & $\%$ & $95 \% C I$ \\
\hline Sensitivity & 66.7 & $9.4-99.2$ & 28.6 & $3.7-71.0$ & 100 & $15.8-100$ \\
\hline Specificity & 97.1 & $89.8-99.6$ & 96.6 & $88.3-99.6$ & 97.1 & $89.9-99.6$ \\
\hline PPV & 50 & $6.8-93.2$ & 50 & $6.8-93.2$ & 50 & $6.7-93.2$ \\
\hline NPV & 98.5 & $92.0-100$ & 91.9 & $82.2-97.3$ & 100 & $94.6-100$ \\
\hline Diagnostic accuracy & 95.8 & $88.1-99.1$ & 89.4 & $79.4-95.6$ & 97.2 & $90.2-99.7$ \\
\hline
\end{tabular}

$C I$, Confidence interval; $P P V$, positive predictive value; $N P V$, negative predictive value.

Hand. An abnormal modified Allen test result was found in 5.6\% (4/71) of the patients. The distribution of the flow patterns of the UA, the SPA, and the TA is summarized in Table I. In the group of 3 patients who had a no-flow pattern in the UA, 1 had a high division of the brachial artery, 1 had calcification of the UA, and the other had an occluded UA at the wrist. The SPA could not be identified in 5 (7\%) patients.

Comparison of the modified Allen test and Doppler ultrasonography dynamic test. The recovery time with the modified Allen test was analyzed with a Box-Cox transformation to satisfy the assumptions of the analysis of variance. For the SPA, UA, and TA flow patterns, analysis of variance indicated a statistically significant difference between the groups (SPA: $\mathrm{F}=$ 7.0, $P<.001$; UA: $\mathrm{F}=13.1, P<.001$; and TA: $\mathrm{F}=8.4$, $P<.001)$ for the modified Allen test. The Tukey multiple comparisons procedure showed that the recovery time by modified Allen test in the no-flow group was significantly different from each of the decreased, increased, and reversed flow groups $(P<.02)$ for all 3 arteries. However, the recovery times in the decreased, increased, and reversed flow groups were not significantly different from each other $(P>0.4$ for all pairwise comparisons).

The no-flow pattern in the Doppler ultrasonography dynamic test was defined as abnormal, and the others were defined as normal. Table II shows the comparison of the modified Allen test results with the Doppler ultrasonography dynamic test in the UA, SPA, and TA groups. Table III represents a summary of the test characteristics of the modified Allen test compared with the Doppler ultrasonographic dynamic test result.

Clinical outcome. Among the 71 patients, 48 $(67.7 \%)$ had their RAs harvested for coronary artery bypass grafting. The choice of conduit depended on the surgeon's preference and on patient enrollment in other clinical trials. None of the patients who had RA harvesting showed signs of hand ischemia in the immediate postoperative period. All of the patients harvested had modified Allen test recovery times of less than 10 seconds. Among the 3 patients who demonstrated a noflow pattern in the UA when the RA was compressed, 1 patient had his or her RA harvested. However, this case was associated with a high origin of the RA, and only the proximal two thirds of the RA were harvested. Four of 7 patients who had a no-flow pattern in the SPA had RA harvesting. None of these patients had hand ischemia postoperatively. Two patients who had noflow patterns in the TA did not have RA harvesting because they both had modified Allen test recovery times of more than 10 seconds.

\section{Discussion}

Although there has been debate over the reliability of the modified Allen test, it has been used for assessing the hand collateral circulation with some proven efficacy. ${ }^{11}$ It has also been used as a screening test at the Austin \& Repatriation Medical Centre since 1994. Between October 1994 and April 2000, a total of 1657 
RAs were harvested from 1323 patients (mean \pm SD age, $64.4 \pm 10.5$ years). The cutoff point of the recovery time of the modified Allen test used in the selection of these patients for RA harvesting was 10 seconds. Thus far, there have been no ischemic complications of the hand. However, the modified Allen test is a subjective operator/patient-dependent test, and there have been a number of reports of hand ischemia after RA intervention, including RA harvesting. ${ }^{12-16}$ Therefore, we sought to find an objective practical method for assessing the hand collateral circulation.

This study thus also sought to evaluate the utility of Doppler ultrasonography for assessing hand collateral circulation. The advantage of the Doppler ultrasonographic technique over other tests is that it can demonstrate the anatomy, measure the flow velocity, and assess the physiologic adaptation of vessels by observing the direction of the blood flow after RA compression. However, the methods of testing and criteria for evaluation vary considerably.

In 1973, Mozersky and colleagues ${ }^{8}$ assessed the completeness of the superficial palmar arch in 70 normal volunteers (140 hands) by using a Doppler ultrasonographic velocity detector. They defined the superficial palmar arch as the most distal transverse vessel in the palm. By observing the changing direction and quantity of the flow velocity when the artery was compressed, they were able to identify a complete arch $(65.7 \%)$ and an incomplete arch (34.3\%). Of 140 hands, ulnar dominant collateral circulation was found in $87.1 \%$, whereas a radial dominant complete arch with good retrograde flow was found in $2.1 \%$. Therefore, in this series $89.2 \%$ of hands were expected to retain adequate circulation when the RAs were occluded.

Doscher and colleagues, in $1983^{9}$ and $1985,{ }^{10}$ examined 100 asymptomatic volunteers (200 hands) with Doppler ultrasonography. The superficial palmar arch was scanned at the same position as that in the study by Mozersky and colleagues. ${ }^{8}$ In most instances RA occlusion produced an increase in the ulnar to radial velocity. This finding was interpreted as a complete superficial palmar arch. When RA compression failed to produce a change in velocity, this was interpreted as an incomplete superficial palmar arch. The incidence of a physiologically incomplete superficial palmar arch in this study was $11 \%$.

In 1976, Kamienski and Barnes ${ }^{17}$ used a Doppler ultrasonographic velocity detector for assessing the continuity of the palmar arch. There were 2 criteria used for defining the continuity. First, the normal arterial velocity signal was described as multiphasic, with a prominent systolic component and one or more diastolic sounds.
An arterial obstruction results in distal arterial velocity signals, which are attenuated with a resultant decrease in the systolic component and loss of normal diastolic sounds. Second, the arterial velocity is increased in response to compression of the opposite artery at the wrist in normal subjects. Using these abnormal criteria of Doppler ultrasonography, the modified Allen test showed complete concordance with the findings obtained with Doppler ultrasonography. However, in our study the ulnar velocity was found to be decreased in 13 (18.3\%) patients. Of these 13 patients, 6 had RA harvesting without ischemic complications. We therefore considered both decreasing and increasing flow velocity in the UA to be normal. These findings emphasize the importance of scanning multiple sites.

Pola and colleagues, ${ }^{1}$ in 1996 , established criteria for an abnormal Doppler ultrasonography dynamic test result to determine which RAs could be harvested. These criteria included patients without an increase in blood flow velocity in the UA associated with flow disappearance in the SPA during RA compression. In this study $5.9 \%$ had an abnormal Doppler ultrasonographic dynamic test result.

Finally, in 1999, Kochi and colleagues ${ }^{2}$ suggested the snuffbox technique. This technique involves examining the RA in the anatomic snuffbox area by using color Doppler ultrasonography. In this study all 10 normal volunteers demonstrated reversed flow in the RA with RA compression. However, our study was more concerned with assessing TA flow and with patients who might have digital arterial diseases.

In our study we first examined the bifurcation point of the brachial artery to confirm the patency of the RAs and UAs in the forearm and the UA at the wrist. Then we measured the flow velocity at 3 different sites: (1) the UA, to assess the inflow of blood supply after RA harvesting; (2) the SPA, which is the terminal branch of the RA at the palmar side; and (3) the TA, which supplies the area of the hand at most risk of ischemia after RA harvesting. The complex patterns of anastomoses among these arteries give rise to a wide range of responses to RA compression in the different hand arteries. This makes interpretation of results more difficult; also, the small size of vessels in the hand can make the detection of arterial flow difficult. The area that was particularly difficult to scan was the SPA. Accordingly, we could not demonstrate this vessel in $7 \%$ (5/71) of cases. This is consistent with the finding in anatomic dissection studies that this vessel is sometimes very small. ${ }^{18}$

In terms of the direction of blood flow with RA compression, our study showed increased flow in the UA in the majority of cases. This indicated a functional conti- 
nuity between the radial and ulnar circulation in the hand. With the RA compressed, the distal perfusion pressure of the capillary bed is reduced. This results in an increase in the pressure gradient between the UA and the capillary bed, causing an increase in UA flow. The reasons for reduced or absent ulnar flow with RA compression are less clear. It is possible that other arteries, such as the median and interosseous arteries, take over from the RA, thereby diverting blood away from the distal UA. To minimize the risk of hand ischemia after RA harvesting, we considered no flow in the UA with RA compression as an abnormal result. Before harvesting the RA in this group, an adequate collateral blood supply should be demonstrated.

The most common flow pattern in the SPA was decreased flow. This can be explained by the reduced perfusion pressure beyond the point of compression of the RA, the SPA's main source of inflow. Increased flow suggests the presence of a large collateral supply flowing in the same direction as the RA. Reversed flow indicates that the RA flow has been replaced by a collateral supply flowing in the opposite direction. However, regardless of the direction of the flow, blood flow was still maintained by collateral flow after RA compression, indicating continuity between the arteries in the hand. Given their potential risk of hand ischemia after RA harvesting, the group of most concern here are those patients who demonstrated a no flow pattern. Accordingly, we considered this as an abnormal test result because it might indicate incomplete superficial and deep palmar arches. Despite this risk, in this study 4 patients with no flow in the SPA with RA compression had RA harvesting without hand ischemia postoperatively. No flow in the SPA with RA compression could also be due to the presence of balanced perfusion pressure at each end of the SPA or a watershed effect through the deep and superficial palmar arch.

The greatest area at risk of ischemia after RA harvesting is the thumb. In our opinion the presence of no flow in the TA represents an absolute contraindication for RA harvesting. Even though some collateral blood supply may develop postoperatively, this group of patients carries a very high risk of hand ischemia.

Comparing the modified Allen test with Doppler ultrasonography, the Allen test accurately predicts Doppler ultrasonographic flow patterns, particularly in the TA. Even though there were only 2 patients in the no flow group of the TA, there was a highly significant correlation with the modified Allen test. Comparing the modified Allen test result with the flow pattern in the TA, the false-positive rate was only $2.9 \%$. Furthermore, no patients with a normal modified Allen test result had no flow in the TA with RA compression. Overall, this study supports the validity of the use of the modified Allen test as a primary screening test for assessing the hand collateral circulation. Doppler ultrasonography is a useful tool for assessing hands that produce an abnormal modified Allen test result but have a good collateral circulation, therefore increasing the number of candidates for RA harvesting.

\section{Conclusion}

The absence of flow in the TA during RA compression is an absolute contraindication to RA harvesting. Absent flow in the SPA and UA is not an absolute contraindication but requires the demonstration of adequate digital artery blood flow before RA harvesting. Doppler ultrasonography of the forearm is useful to exclude patients whose UA is absent, hypoplastic, or diseased.

An increased recovery time with the modified Allen test predicts absence of flow in the TA in Doppler ultrasonographic flow patterns, demonstrating its validity as a primary screening test. The use of the Doppler ultrasonography dynamic test in conjunction with the Allen test permits the safe harvesting of some RAs in patients with a false-positive abnormal modified Allen test.

We thank the cardiac surgeons at the Austin \& Repatriation Medical Centre (Drs George Matalanis, Alexander Rosalion, and Jai Raman) for their cooperation and help and Dr Tania Lewis for her editorial assistance.

Received for publication Aug 23, 2000; revisions requested Sept 7, 2000; revisions received Oct 13, 2000; accepted for publication Oct 20, 2000.

Address for reprints: Brian F. Buxton, MS, FRACS, Department of Cardiac Surgery, Austin Campus, HSB-5, Austin \& Repatriation Medical Centre, Studley Rd, Heidelberg, Victoria 3084, Australia (E-mail: bux@austin.unimelb.edu.au).

\section{REFERENCES}

1. Pola P, Serricchio M, Flore R, Manasse E, Favuzzi A, Possati GF. Safe removal of the radial artery for myocardial revascularization: a Doppler study to prevent ischemic complications to the hand. J Thorac Cardiovasc Surg 1996;112:737-44.

2. Kochi K, Sueda T, Orihashi K, Matsuura Y. New noninvasive test alternative to Allen's test: snuff-box technique. J Thorac Cardiovasc Surg 1999;118:756-8.

3. Johnson WH III, Cromartie RS III, Arrants JE, Wuamett JD, Holt JB. Simplified method for candidate selection for radial artery harvesting. Ann Thorac Surg 1998;65:1167.

4. Fuhrman TM, McSweeney E. Noninvasive evaluation of the collateral circulation to the hand. Acad Emerg Med 1995;2:195-9.

5. Mercier FJ, Basdevant C, De Tovar G, Fischler M. Doppler preoperative evaluation of the prevalence of functional abnormalities of palmar arches in children. Ann Fr Anesth Reanim 1994; 13:785-8. 
6. Ruland O, Borkenhagen N, Prien T. The Doppler palm test. Ultraschall Med 1988;9:63-6.

7. Marcillon M, Maestracci P, Guillot F, Dulbecco P, Filippi C, Valici A. Doppler velocimetric evaluation of the reliability of Allen's test for radial artery catheterization. Ann Fr Anesth Reanim 1982;1:403-6.

8. Mozersky DJ, Buckley CJ, Hagood CO Jr, Capps WF Jr, Dannemiller FJ Jr. Ultrasonic evaluation of the palmar circulation. Am J Surg 1973;126:810-2.

9. Doscher W, Viswanathan B, Stein T, Margolis IB. Hemodynamic assessment of the circulation in 200 normal hands. Ann Surg 1983;198:776-9.

10. Doscher W, Viswanathan B, Stein T, Margolis IB. Physiologic anatomy of the palmar circulation in 200 normal hands. J Cardiovasc Surg 1985;26:171-4.

11. Husum B, Berthelsen P. Allen's test and systolic arterial pressure in the thumb. Br J Anaeth 1981;53:635-7.

12. Nunoo-Mensah J. An unexpected complication after harvesting of the radial artery for coronary artery bypass grafting. Ann Thorac Surg 1998;66:929-31.

13. Valji K, Hye RJ, Roberts AC, Oglevie SB, Ziegler T, Bookstein $\mathrm{JJ}$. Hand ischemia in patients with hemodialysis access grafts: angiographic diagnosis and treatment. Radiology 1995;196:697-701.

14. Jones BM, O'Brien CJ. Acute ischemia of the hand resulting from elevation of a radial forearm flap. $\mathrm{Br} \mathrm{J}$ Plast Surg 1985;38:396-7.

15. Crossland SG, Neviaser RJ. Complications of radial artery catheterization. Hand 1977;9:287-90.

16. Baker RJ, Chunprapaph B, Nyhus LM. Severe ischemia of hand following radial artery catheterization. Surgery 1976;80:449-57.

17. Kamienski RW, Barnes RW. Critique of the Allen test for continuity of the palmar arch assessed by Doppler ultrasound. Surg Gynecol Obstet 1976;142:861-4.

18. Coleman SS, Anson BJ. Arterial patterns in the hand based upon a study of 650 specimens. Surg Gynecol Obstet 1961;113:409-24. 